\title{
Whole Genome Analysis and MicroRNAs Regulation in HepG2 Cells Exposed to Cadmium
}

\author{
Marco Fabbri ${ }^{1 *}$, Chiara Urani ${ }^{2 *}$, Maria Grazia Sacco ${ }^{1}$, \\ Claudio Procaccianti ${ }^{2}$, and Laura Gribaldo ${ }^{1}$ \\ ${ }^{1}$ Institute for Health and Consumer Protection, Molecular Biology and Genomics Unit, Joint Research Centre, Ispra (VA), Italy; \\ ${ }^{2}$ Environmental Sciences Department, University of Milano Bicocca, Milano, Italy
}

\begin{abstract}
Summary
Cadmium $(C d)$ is a metal known to be toxic and carcinogenic, but its mechanism of action remains to

be fully elucidated.

We investigated the gene expression modulation in the human hepatoma cell line HepG2 after exposure

to $2 \mu \mathrm{M}$ and $10 \mu \mathrm{M} C d$ using an Agilent microarray. Furthermore, we evaluated the microRNA modulation

after exposure to $10 \mu \mathrm{MCd}$ with a Low Density Array.

At the low concentration only eleven genes belonging to the metallothionein familiy were regulated.

At the higher concentration the pathway enrichment analysis for the 536 up-regulated genes showed a

large number of pathways related to cancer, whereas the 424 down-regulated genes were enriched

on pathways correlated to liver function. A large percentage of modified microRNAs belonged to the

let-7 family, which is considered to have oncosuppressor functions.

Several pathways connected to cancer were regulated at the transcription level, and miRNAs had

a potential impact on the modulation of this regulation.
\end{abstract}

Keywords: cadmium, gene ontology, HepG2 cells, pathway mapping, toxicogenomics

\section{Introduction}

Cadmium is a naturally occurring metal continuously released into the atmosphere by burning fossil fuels. Due to its physical and chemical properties, it has a wide range of applications (e.g., from manufacturing to agricultural activities) and thus raises serious concern, both for the environment and for human health.

Routes for non-occupational $\mathrm{Cd}$ exposure in humans are mainly via ingestion of contaminated food and water, as well as by inhalation, with active and passive smokers subject to inhaling particularly high doses (Talio et al., 2010).

As a toxic metal, $\mathrm{Cd}$ is unlikely to enter animal cells by specific mechanisms, but its uptake is mediated by the L-type calcium channel (LTCC) (Ohana et al., 2006) and by protonmetal co-transporters (e.g., DMT1, divalent metal transporter-1) (Martelli et al., 2006). Once it crosses the membrane barrier, Cd accumulates primarily within kidneys and liver, and it can pass through alveolar cells to enter the blood. $\mathrm{Cd}$ is poorly excreted and cannot undergo metabolic degradation to less toxic species, leading to long-term storage and an accumulation in the higher micromolar range in tissues of non-occupationally exposed populations (Hartwig et al., 2002). The extremely long biologi- cal half-life (20-30 years) is attributable, in part, to a family of metal-binding proteins, the metallothioneins (MT), which tightly bind the metal and have evolved as a defense system to protect against metal reactivity with macromolecules.

Molecular aspects of $\mathrm{Cd}$ toxicity have been recently reviewed (Martelli et al., 2006; Bertin and Averbeck, 2006; Hartwig, 2010) and range from thiol-containing protein affection and consequent production of reactive oxygen species, to changes in gene expression triggering cell cycle arrest, apoptosis, differentiation, and immortalization. $\mathrm{Cd}$ and $\mathrm{Cd}$ compounds have been classified as carcinogenic to humans (Group 1) by the World Health Organization's International Agency for Research on Cancer (IARC, 1993), acting as epigenetic or indirect genotoxic compounds. The most persuasive data for $\mathrm{Cd}$ carcinogenicity comes from epidemiological studies relating lung cancer to occupational inhalation of the metal (Waalkes, 2003).

Carcinogenesis is a multi-step process, and the expression of the final phenotype could be the effect of a complex of events including, among others, the deregulation of checkpoints and cell control systems. One of the mechanisms that is promptly involved in DNA-damaged cells to avoid the propagation of harmful stimuli is the activation of the tumor suppressor protein $\mathrm{p} 53$.

* Marco Fabbri and Chiara Urani contributed equally to this work

Received November 29, 2011; accepted in revised form March 8, 2012. 
The $\mathrm{p} 53$ protein is one crucial transcription factor whose activity may be impaired by $\mathrm{Cd}$ exposure with suppression of p53-mediated cell cycle arrest in response to DNA damage. Interference with DNA repair systems or their inhibition, as previously reported in Cd-exposed cells, may lead to possible propagation of genetic damage. Furthermore, critical steps in the carcinogenesis process, and particularly in cancer invasion and metastasis, are structural and morphological changes that involve the actin machinery and highly organized membrane protrusions, the focal adhesions, podosomes, and invadopodia (Albiges-Rizo et al., 2009). Particular attention should be paid to these dynamic, actin-rich structures, as well as to the degradation of the extracellular matrix, which could play a role in $\mathrm{Cd}$ carcinogenesis.

However, even though a huge number of published papers concerning $\mathrm{Cd}$ effects is available in various model systems, from bacteria to mammals, the mechanism of action of this metal, especially concerning its carcinogenicity, is not yet fully understood.

Toxicogenomics through microarray technology is a valid tool for understanding the complex regulation circuitry within the cells and specific pathways triggered by $\mathrm{Cd}$ exposure. A DNA microarray enables large scale simultaneous detection of gene regulation, allowing a mechanism-based classification of environmental contaminants. This framework is further complicated by the recent findings on microRNA (miRNA) functions. These widely distributed molecules are small RNAs (18-25 nucleotide long) that play important gene-regulatory roles targeting both messenger RNA (mRNA) degradation and suppression of protein translation, therefore exhibiting modulating functions on protein-coding genes and regulating multiple cellular processes, including proliferation and apoptosis (Bartel, 2009).

An increasing number of toxicogenomic studies (Ao et al., 2010; Badisa et al., 2008; Honda et al., 2010; Kawata et al., 2009; Koizumi and Yamada, 2003) has described the specific gene expression pattern in normal animal tissues and cultured cells, or transformed ones exposed to metals (e.g., Cd, As, Ni). The microarray technology also has been applied to gene expression signature and risk assessment, e.g., in a Japanese population environmentally exposed to Cd (Dakeshita et al., 2009). Nevertheless, all studies have analyzed a relatively reduced number of genes, from tens to a few thousand, centering the attention on specific regulated pathways (e.g., protective functions, cell cycle, apoptosis) or comparing molecular mechanisms triggered by $\mathrm{Cd}$ to those triggered by known carcinogens.

In the current work, we studied the expression of genes and miRNAs modulated by $\mathrm{Cd}$. For this purpose, a whole-genome analysis by cDNA microarray was performed in the human hepatoma cell line HepG2, after 24 h exposure to low (2 and 10 $\mu \mathrm{M}$ ), human-relevant Cd concentrations (Andrew et al., 2003; Satarug and Moore, 2004). A microRNA analysis was further performed to clarify their roles as key mediators of the cellular response to this environmental contaminant. Our results provide a better understanding of the complex mechanisms of regulation and, as a consequence, of the effects of this carcinogenic metal. Toxicogenomics is a valid tool when using alternative testing models for the assessment of toxicity. The combined evaluation of mRNA and microRNA expression allowed us to iden- tify a possible mode of action of Cd. Furthermore, we identified pathways and microRNA regulated by $\mathrm{Cd}$ that could be used as biomarkers, thus avoiding animal testing.

\section{Materials and methods}

\section{Cell culture and treatment}

Human hepatoblastoma cells (HepG2) were used, as they represent a useful in vitro model for cadmium toxicity studies (Dehn et al., 2004; Urani et al., 2005, 2007, 2010). HepG2 cells were routinely cultured in Opti-MEM medium (Invitrogen, San Giuliano Milanese, Italy), supplemented with $10 \%$ fetal bovine serum, and $1 \%$ antibiotics. Cells were kept in an incubator at $37^{\circ} \mathrm{C}$ under a humidified $5 \% \mathrm{CO}_{2}$ atmosphere.

Viability of HepG2 cells exposed to the highest $\mathrm{CdCl}_{2}$ (Sigma-Aldrich, Milan, Italy) concentration $(10 \mu \mathrm{M})$ was between $80-90 \%$, as previously demonstrated by MTT test; the computed IC 50 value was $25.5 \mu \mathrm{M}$ (Urani et al., 2005).

For RNA and miRNAs expression profiling, cells were plated on day 0 onto $100 \mathrm{~mm}$ diameter Petri dishes (Corning) at a density of $1.5 \times 10^{6}$ cells/dish. Twenty-four hours after seeding (day 1), the medium was removed and the cells were either treated with fresh medium added with $\mathrm{CdCl}_{2}(\mathrm{Cd})$ at a density of $2 \mu \mathrm{M}$ and $10 \mu \mathrm{M}$, or with complete medium (control) for 24 h (day 2).

\section{Treatments and RNA extraction}

After $24 \mathrm{~h}$ of treatment (day 2), the cells were washed with cold PBS and lysed in $350 \mu \mathrm{l}$ RLT lysis buffer (Qiagen, Germantown, MD, USA) following the manufacturer's instructions. Samples were stored at $-80^{\circ} \mathrm{C}$ until RNA extraction was carried out. Three independent replicates of the experiments were performed.

For miRNAs extraction, the cells were plated on day 0 and treated with $10 \mu \mathrm{M} \mathrm{CdCl}_{2}$ on day 1 , as described. On day 2, cells were washed in cold PBS and lysed in Mirvana lysis buffer (Ambion), according to the manufacturer's instructions. Samples were stored at $-80^{\circ} \mathrm{C}$ until RNA extraction was carried out. Two independent replicates of the experiment were performed.

\section{Microarray expression profiling}

RNA was purified from HepG2 cells using the RNeasy Plus kit (Qiagen, Germantown, MD, USA). RNA was quantified using a ND-1000 UV-Vis Spectrophotometer (NanoDrop Technologies), and the integrity of the RNA was assessed with the Agilent 2100 Bioanalyzer (Agilent), according to the manufacturer's instructions. RNA samples used in this study all had a 260/280 ratio above 1.9 and a RNA Integrity Number (RIN) above 9.0.

The microarray experiment was designed to perform three biological replicates for each treatment. All sample-labeling, hybridization, washing, and scanning steps were conducted following the manufacturer's specifications. In brief, Cy3-labeled cRNA was generated from 500 ng input total RNA using Quick Amp Labeling Kit, One-color (Agilent).

For every sample, $1.65 \mu \mathrm{g}$ cRNA from each labeling reaction (with a specific activity above 9.0) was hybridized using 
the Gene Expression Hybridization Kit (Agilent) to the Agilent Whole Human Genome Oligo Microarray (Agilent), which is a 4 x 44k 60mer slide format where each of the 4 arrays represents about 41,000 unique genes and transcripts. After hybridization, the slides were washed and then scanned with the Agilent G2565BA Microarray Scanner (Agilent). The fluorescence intensities on scanned images were extracted and preprocessed by Agilent Feature Extraction Software (v10.5.1.1).

Quality control and array normalization was performed in the $\mathrm{R}$ statistical environment using the Agi4x44PreProcess package downloaded from the Bioconductor web site (Gentleman et al., 2004). The normalization and filtering steps were based on those described in the Agi4x44PreProcess reference manual.

Principal-component analysis (PCA) was carried out on all genes under investigation to determine expression trends within the dataset. PCA is a useful technique to reduce the dimensionality of large data sets.

In order to detect expression differences among different cell populations a moderate $t$ test was applied. Moderated t statistics were generated by the Limma Bioconductor package. Modulated genes were chosen as those with a fold change greater than 2 and a false discovery rate (Benjamini and Hochberg's method) corrected p-value smaller than 0.05 (Smyth, 2004). All of the above computations were conducted using the R statistics programming environment.

Up-regulated and down-regulated genes were analyzed in the KEGG database to identify genes with similar functions. Expression Analysis Systematic Explorer (EASE) biological theme analysis was conducted online using DAVID (Huang et al., 2009).

Microarray data have been submitted to NCBI's Gene Expression Omnibus repository (Edgar et al., 2002) and are available under accession number GSE31286.

\section{Real-time PCR validation of microarray}

A real-time PCR analysis was done on the RNA samples used for microarray hybridization, with the aim of validating the results. The RNA samples were reverse-transcribed using the Taqman High Capacity Reverse Transcription Kit (Applied Biosystems) in $20 \mu \mathrm{l}$ reactions containing $1 \mu \mathrm{g}$ total RNA according to the manufacturer's instructions. The qPCR reactions containing 50 ng RNA equivalents were performed in optical 96-well plates using an ABI 7900HT Sequence Detection System (Applied Biosystems). Pre-designed Taqman assays were supplied as $20 \mathrm{X}$ primer-probes mixes by Applied Biosystems. PCR reactions were prepared using Taqman Universal PCR Master Mix (Applied Biosystems). The following Taqman assays were used: CAPN2-Hs00965092_m1, COL1A1-Hs01076775_g1, FOS-Hs01119267_g1, GADD45B-Hs00169587_m1, HSPA6Hs00275682_s1,ITGB1-Hs01127547_m1,ITGA2-Hs00158148_ m1, ITGA3-Hs00233722_m1, LAMB3-Hs00165078_m1, JUNHs00277190_s1, and HPRT1-Hs99999909_m1.

The following reaction conditions were used: $95^{\circ} \mathrm{C}$ for 10 min, followed by 40 cycles of $95^{\circ} \mathrm{C}$ for $15 \mathrm{sec}$ and $60^{\circ} \mathrm{C}$ for $60 \mathrm{sec}$. Duplicate reactions were performed for each sample. Baseline fluorescence and threshold were set automatically using SDS 2.3 software (Applied Biosystems). Data were fur- ther analyzed in Microsoft Excel (2003) using the deltadeltaCt method(HPRT1-Hs99999909_m1 as reference gene).

\section{MicroRNA expression profiling}

RNA was extracted using the MIRVANA kit (AMBION) according to the manufacturer's instructions. This protocol allows the isolation of total RNA enriched with miRNAs. RNA concentration and quality were determined by Nanodrop and 2100 Agilent Bioanalizer. The RIN was always above 8.5.

Total RNA was reverse transcribed with Taqman MicroRNA Reverse Transcription Kit using Megaplex ${ }^{\text {TM }}$ RT Primers (Applied Biosystems). Real-time PCR reactions were carried out on pre-configured microfluidic cards (Taqman Array MicroRNA Cards, set A,V2.2 and set B, V3, Applied Biosystems) allowing the detection of about 754 unique specific assays and four candidate endogenous control assays.

Two biological replicates for control and two for $10 \mu \mathrm{M} \mathrm{Cd}$ were tested. Experimental data were then analyzed by SDS 2.3 software (Applied Biosystems) and the relative expression values were calculated using as endogenous control U6 for miRNA. MiRNAs with a threshold cycle $<33$ that showed a fold change $>4$ in samples treated with $\mathrm{Cd}$ as compared to control samples were considered differentially expressed.

The Diana MirPath application was used to identify pathways enriched in the predicted targets for regulated miRNAs (Boyerinas et al., 2010). In the MirPath application for miRNA target prediction, the Diana microT 4.0 algorithm based on Artificial Neural Networks was used (Maragkakis et al., 2009).

\section{Results}

\section{Whole genome analysis}

We carried out a principal component analysis (PCA) to find trends in the regulation of genes caused by $\mathrm{Cd}$ exposure. PCA was used to map the entire dataset on a two axis graph (principal components, PC1 and PC2) where the distances account for similarity (Fig. 1). A clear, dose-dependent $\mathrm{Cd}$ gene regulation was observed on the first principal component (PC1), explaining $33.8 \%$ of the sample variance, with $10 \mu \mathrm{M} \mathrm{Cd}$ strongly stimulating the change in gene expression, while $2 \mu \mathrm{M} \mathrm{Cd}$ and control data were closer to each other.

The eleven genes regulated (fold induction greater than 2 and a p-value smaller than 0.05 ) by $2 \mu \mathrm{M} \mathrm{Cd}$ were up-regulated (Fig. 2). All belong to the family of metallothioneins, proteins that bind to zinc and cadmium and protect against metal exposure and oxidative stress (Table of Fig. 2).

The higher Cd concentration produced a regulation of 960 genes, 536 up-regulated and 424 down-regulated (Fig. 2). The two groups (up- and down-regulated) were analyzed separately for pathway enrichment. As shown in Table S1 (Supplementary file at www.altex-edition.org), the down-regulated genes were enriched in pathways that represent typical functions of liver. We found 22 out of 68 genes regulated in the pathway of complement and coagulation cascades (Fig. 3), and 11 out of 39 genes regulated in the pathway of fatty acid metabolism (data not shown), so we can argue that the toxic effect of $\mathrm{Cd}$ in- 


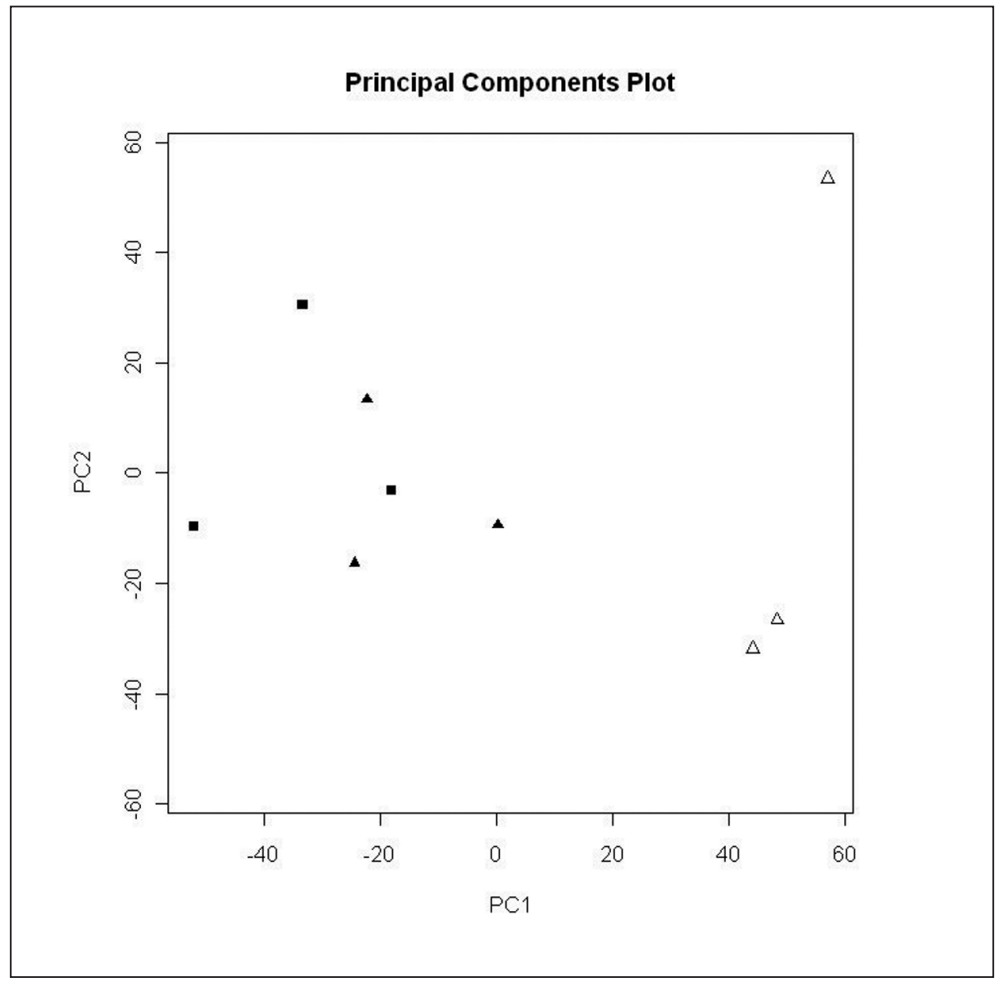

Fig. 1: Principal component analysis of the transcriptome

PCA was carried out on all genes under investigation to determine expression trends within the dataset. A sample trend is shown in a scatter plot of the principal components PC1 and PC2. The first component accounts for $33.8 \%$ and the second for $25.2 \%$ of the system variance. Black triangles represent $2 \mu \mathrm{M} \mathrm{Cd}$, white triangles $10 \mu \mathrm{M} \mathrm{Cd}$ and black squares represent control.

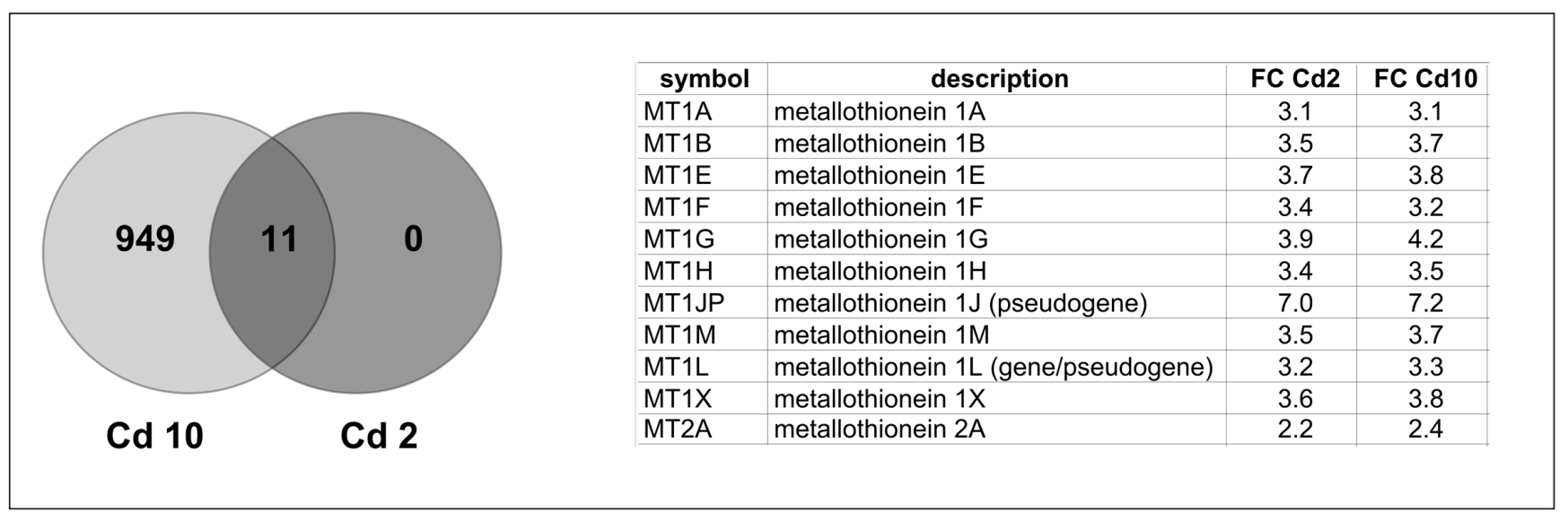

Fig. 2: Venn diagram showing the number of regulated genes

The Venn diagram represents genes regulated by $2 \mu \mathrm{m}$ and $10 \mu \mathrm{m} \mathrm{Cd}$. The eleven genes regulated in the same direction by both stimuli are summarized in the Table. FC, fold change.

duces a reduction of the main liver functions. The up-regulated genes (Tab. S2, supplementary file at www.altex-edition.org) were enriched in pathways related to cancer and inflammation, in agreement with the IARC classification of $\mathrm{Cd}$ as a human carcinogen. A detail of the focal adhesion pathway, related to cancer, is provided (Fig. 4).

Real-time PCR validation of microarray

A subset of regulated genes previously identified for their relevance were validated with real-time RT-PCR (qPCR). Consistently with the array data, the expression of CAPN2 (calpain 2), COL1A1 (collagen, type I, alpha 1), FOS (FBJ murine osteosar- coma), GADD45B (growth arrest and DNA-damage-inducible, beta), HSPA6 (heat shock 70kDa protein 6), ITGA2 (integrin, alpha 2), ITGA3 (integrin, alpha 3), ITGB1 (integrin, beta 1), LAMB3 (laminin, beta 3), JUN (jun proto-oncogene) was induced by $10 \mu \mathrm{M}$ cadmium. The trends of all validated genes were confirmed by qPCR (Tab. 1A).

\section{MicroRNAs expression profiling}

To further understand the regulatory activities of $\mathrm{Cd}$, we investigated the expression of miRNAs, small noncoding RNAs capable of regulating gene expression at the translational level. 


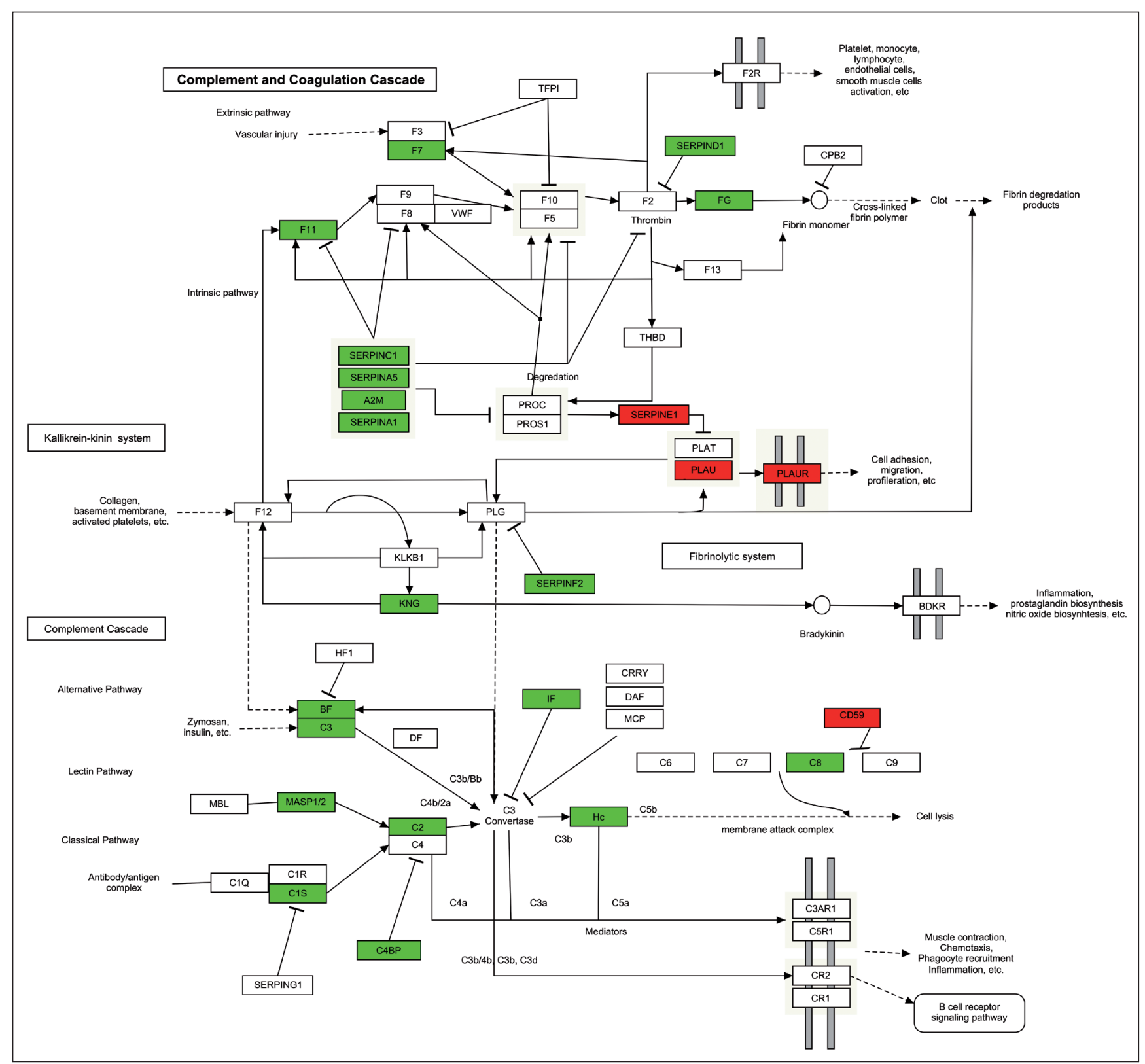

Fig. 3: Complement and coagulation KEGG map

Representation of the KEGG map for complement and coagulation cascades. Genes in red are up-regulated, in green down-regulated.

The effects of $10 \mu \mathrm{M}$ Cd on miRNAs were evaluated with microfluidic cards. Four of the regulated miRNAs (4 out of 12) belong to the let-7 family (Tab. 1B). The miRNAs belonging to this family have been described as tumor suppressors and, in agreement with their function, these miRNAs are reported to be down-regulated in many cancers (Boyerinas et al., 2010).

In order to identify potential pathways altered by the miRNAs, regulated miRNAs were analyzed with the DIANA-mirpath algorithm. This software, starting from a set of miRNAs, performs an enrichment analysis of predicted targets, giving indications of possible functions of the regulated miRNAs ( $\mathrm{Pa}-$ padopoulos et al., 2009).
The highly scored target pathways predicted by the software (Tab. S3, supplementary file at www.altex-edition.org) are focal adhesions and the mitogen-activated protein kinase (MAPK) that are involved in regulation of proliferation and differentiation processes related to cancer. Other top predicted pathways are also associated with cancer: prostate cancer, glioma, and the p53 signaling pathway. Furthermore, the regulation of actin cytoskeleton, a known quantitative marker for cellular transformation (Rao et al., 1990), has been scored as target pathway as well. This gives a clear prediction of an activity of tumor suppressors for those miRNAs, and their down regulation can create a molecular environment prone to the development of cancer. 


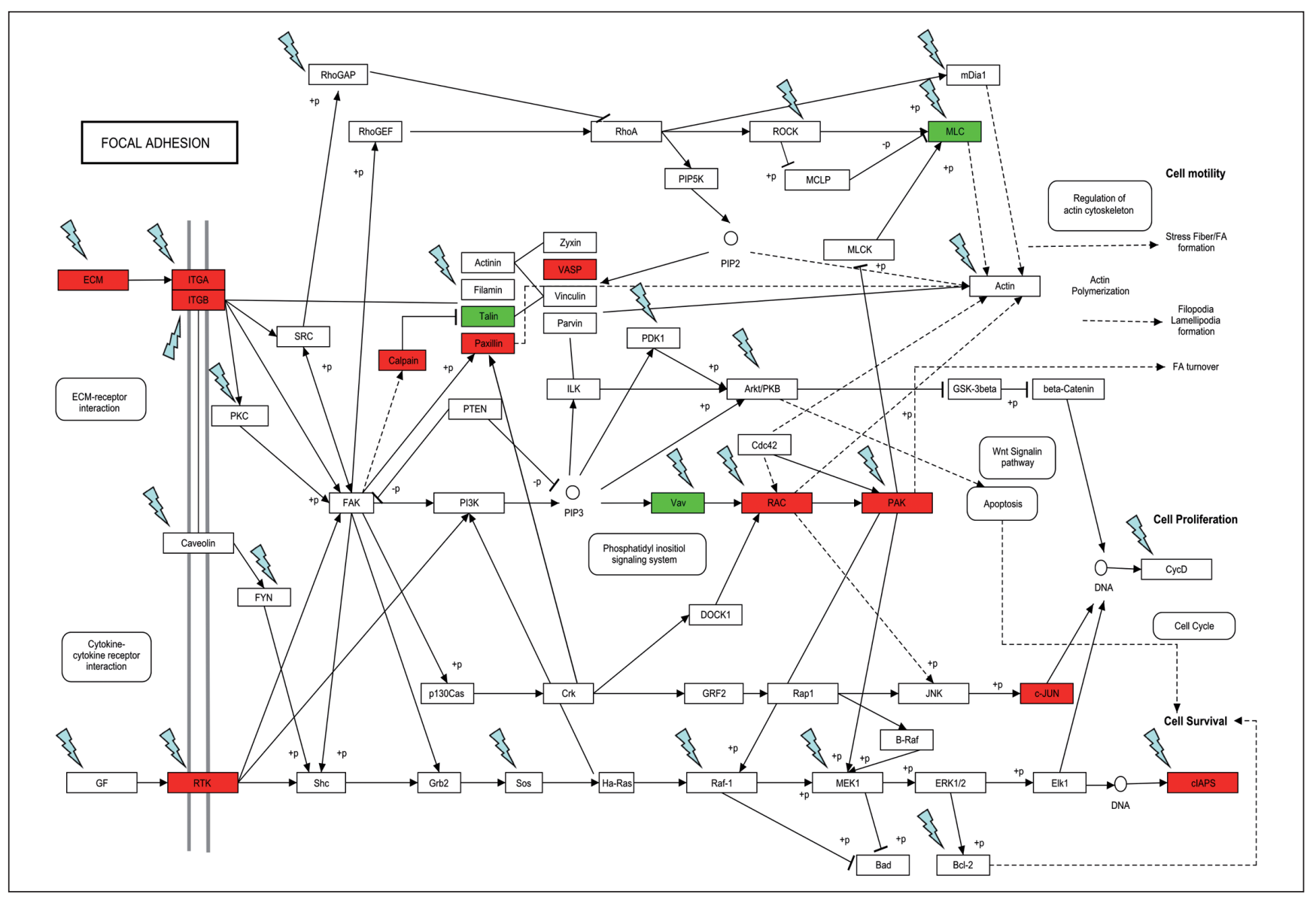

Fig. 4: Focal adhesion KEGG map

Representation of the KEGG map for focal adhesion. Genes in red are up-regulated, in green down-regulated, flash symbol indicates genes predicted by Mirpath as targets of our sets of down-regulated miRNAs.

Tab. 1: Validation of selected regulated genes

(A) Validation of a selection of genes regulated by $10 \mu \mathrm{M}$ Cd. "FC qPCR" column shows the regulation measured by real-time PCR technology and "FC Array" column shows the measurement done with microarray technology. Fold change (FC) expresses the difference of the mean log control and mean log Cd stimulated data; (B) miRNAs regulated by $10 \mu \mathrm{M}$ Cd with fold greater than 4. Fold change (FC) expresses the difference of the mean log control and mean log Cd stimulated data.

A

\begin{tabular}{|l|l|l|}
\hline Gene & FC qPCR & FC Array \\
\hline CAPN2 & 1.56 & 2.05 \\
\hline COL1A1 & 2.57 & 1.80 \\
\hline FOS & 4.92 & 2.84 \\
\hline GADD45B & 4.11 & 3.72 \\
\hline HSPA6 & 9.43 & 5.28 \\
\hline ITGA2 & 2.19 & 1.84 \\
\hline ITGA3 & 2.11 & 1.27 \\
\hline ITGB1 & 0.68 & 1.27 \\
\hline JUN & 1.30 & 2.22 \\
\hline LAMB3 & 3.24 & 3.44 \\
\hline
\end{tabular}

B

\begin{tabular}{|l|l|}
\hline Detector & FC \\
\hline hsa-let-7a & -3.41 \\
\hline hsa-let-7b & -2.22 \\
\hline hsa-let-7e & -2.55 \\
\hline hsa-let-7g & -2.09 \\
\hline hsa-miR-1233 & -3.90 \\
\hline hsa-miR-1275 & -2.03 \\
\hline hsa-miR-130a & -3.07 \\
\hline hsa-miR-15b & -2.54 \\
\hline hsa-miR-15b* & -2.40 \\
\hline hsa-miR-23b & -2.97 \\
\hline hsa-miR-361-5p & -2.25 \\
\hline hsa-miR-455-3p & -2.27 \\
\hline
\end{tabular}




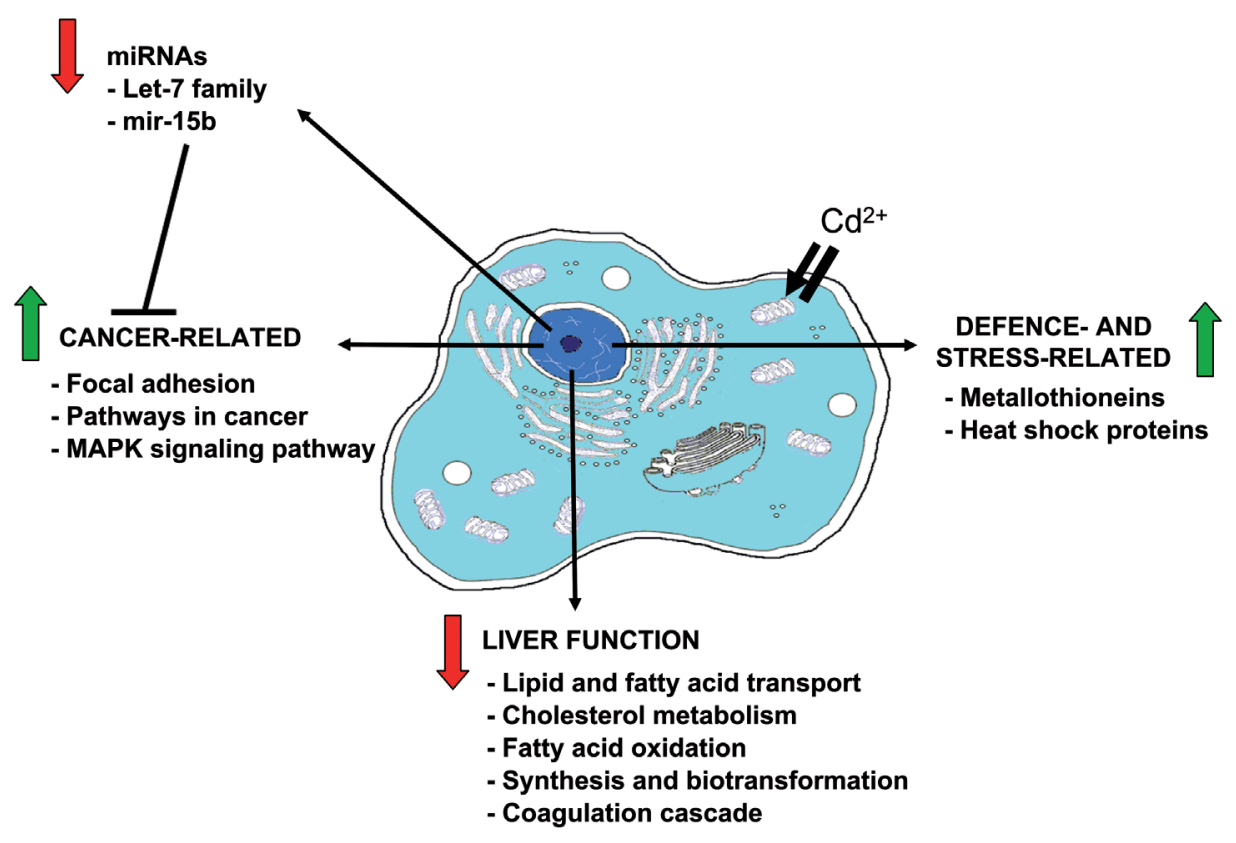

Fig. 5: Schematic model of Cd activity

Schematic representation of $\mathrm{Cd}^{2+}$ uptake by mammalian cells and its effects at molecular level: gene and miRNAs expression. Proven transport pathways of water soluble Cd compounds in mammalian cells are through ion transporters and channels, while particulate $\mathrm{Cd}$ compounds may be taken up by phagocytosis. Once taken up into HepG2 cells, Cd regulates different families of genes including defense- and stress-related (up-regulated), liver function-related (down-regulated), and cancer-related (down-regulated). In addition, the let-7 and 15-b family of miRNAs, are down-regulated, thus leading to a possible deregulation of cancer-related pathways. See the text for details. Green arrow: upregulation; red arrow: downregulation.

\section{Discussion}

Cadmium is a widespread environmental and industrial pollutant. The U.S. Consumer Product Safety Commission (CPSC) has made several recalls due to concerns about levels of $\mathrm{Cd}$ used in red and yellow paints for toys (IARC, 1993).

Cadmium occurs in all food, but agricultural crops (particularly irrigated rice) generally account for most of the intake. A wide range of $\mathrm{Cd}$ effects has been reported at the cellular and molecular level (Martelli et al., 2006; Bertin and Averbeck, 2006; Hartwig, 2010). In some cases the results are controversial, however, and in recent years there has been an ongoing discussion on $\mathrm{Cd}$ general effects and toxicity. In the current work, we studied the expression of genes and metabolic pathways specifically modulated by $\mathrm{Cd}$. For this purpose, a whole-genome analysis by cDNA microarray was performed in the human hepatoma cell line, HepG2, after 24 h exposure to low ( 2 and 10 $\mu \mathrm{M}$ ), human-relevant Cd concentrations (Andrew et al., 2003; Satarug and Moore, 2004). A miRNA analysis was performed to clarify their roles as key mediators of the cellular response to this environmental contaminant.
A general overview of the main gene families modulated by $\mathrm{Cd}$ in HepG2 cells is schematically provided in Figure 5.

The whole gene expression level showed a dose-dependent effect, with all genes regulated by $\mathrm{Cd} 2 \mu \mathrm{M}$ also modulated at $10 \mu \mathrm{M}$ and, in addition, a set of genes (949) specifically regulated by the highest $\mathrm{Cd}$ concentration (Fig. 2). The trends of 10 of those genes also were confirmed by qPCR, validating the microarray approach (Tab. 1A).

Once taken up, $\mathrm{Cd}$ is distributed, particularly to kidneys and the liver, where the production of metallothioneins (MT) is induced. About $80-90 \%$ of $\mathrm{Cd}$ in the body is bound to MT (Klaassen et al., 2009), and the binding of $\mathrm{Cd}$ to MT prevents the free $\mathrm{Cd} 2^{+}$ ions from exerting their toxic effects by interaction with proteins, transcription factors, and nucleic acids. Toxicity may be considered to occur when the binding capacity of MT is surpassed, as previously demonstrated by saturation curves in MT protein expression reached after long-term Cd exposure in HepG2 cells (Urani et al., 2007). In this respect, our data on gene expression confirmed previous findings on the up-regulation of defense- and stress-related members of the MT family by Cd in HepG2 cells (Urani et al., 2005, 2010), as well as in other cell systems (Bon- 
ham et al., 2003). Herewith, eleven members of the MT family are up-regulated with a similar fold induction (Table of Fig. 2) between the two concentrations of Cd used. MT are strongly induced by the metal sensor MTF-1 (metal-regulatory transcription factor 1), a metalloregulatory transcription factor, in response to altered metal ion concentrations (e.g., zinc and cadmium) and oxidative stress (Li et al., 2008) to trigger gene expression for restoring metal homeostasis. Oxidative stress-related reactions and ROS production are likely to be responsible for DNA singlestrand breaks after Cd intoxication, and they appear to be relevant in Cd-induced carcinogenicity (Bertin and Averbeck, 2006). Other relevant stress-related gene members up-regulated by $\mathrm{Cd}$ in this work are the heat shock proteins (hsp). In particular, hsp), in particular hsp70 and hsp22, confirming their role as a universal response to stress, protecting cells from harmful environmental conditions.

In terms of liver function, this target organ plays a key role in lipid metabolism, being the hub of fatty acid synthesis and lipid circulation through lipoprotein synthesis. Eventually, the accumulation of lipid droplets into the hepatocytes results in hepatic steatosis, which may develop as a consequence of multiple dysfunctions such as alterations in beta-oxidation, very low density lipoprotein secretion, and pathways involved in the synthesis of fatty acids. By altering essential metals homeostasis (Martelli et al., 2006), excess cadmium can cause a rise in cholesterol levels. Levels of other fats may be adversely affected if liver function is impaired by cadmium toxicity. In our model of human liver-derived cells, we saw down-modulation of genes involved in lipid and fatty acid transport, cholesterol metabolism, fatty acid oxidation, as well as many substrates of fatty acid synthesis and biotransformation. Moreover, acute liver injury causes a decreased production of proteins such as albumin and blood clotting proteins. We observed modulation both of the classical pathway $\mathrm{C} 1 \mathrm{~s}$ (complement component 1, s subcomponent), C2 (complement component 2), C4BP (complement component 4 binding protein, alpha), C5 (complement component 5), and the intrinsic pathway serpin (serpin peptidase inhibitor) A1, A5, C1, D1, A2M, regulating, ultimately, the coagulation cascade (Fig. 3).

$\mathrm{Cd}$ and $\mathrm{Cd}$ compounds have been classified as carcinogenic to humans (Group 1) by the World Health Organization's International Agency for Research on Cancer (IARC, 1993). They act by multiple indirect mechanisms rather than by direct genotoxicity, as current evidence suggests (Hartwig, 2010). Until now, however, these mechanisms have not been fully understood; they are currently under investigation.

In this regard, on the side of up-modulation, we observed cancer-related families of genes regulated by $\mathrm{Cd}$ exposure, as demonstrated by KEGG enrichment (Tab. S2, supplementary file at www.altex-edition.org), with focal adhesion members: COL1A1 (collagen, type I, alpha 1), COL4A2 (collagen, type IV, alpha 2), BIRC3 (baculoviral IAP repeat-containing 3), ITGA6 (integrin, alpha 6), ITGA2 (integrin, alpha 2), ITGA3 (integrin, alpha 3), ITGB1 (integrin, beta 1), JUN (jun proto-oncogene), LAMB3 (laminin, beta 3), MET (met proto-oncogene), PAK1 (p21 protein activated kinase 1), PXN (paxillin), RAC2 (ras-related C3 botulinum toxin substrate 2), VASP (vasodilator-stimulated phosphoprotein), VTN (vitronectin), and CAPN2 (calpain 2), see Table S4 (Supplementary file at www.altex-edition.org), having highest statistical significance. Focal adhesions are dynamic organelles that play essential roles in important biological processes, including cell motility, proliferation, differentiation, survival, and regulation of gene expression. The regulation of focal adhesion along with the deregulation of the actin cytoskeleton represent motility mechanisms that facilitate invasion and metastasis of cancer cells (Jiang et al., 2009; Lambrechts et al., 2004). Our results show that other up-regulated genes closely related to these processes are integrines, Cdc42 effector protein 2, Rho family GTPase 3, and actin. For focal adhesions, specialized structures like bundles of actin filaments, are anchored to transmembrane receptors of the integrin family through a multi-molecular complex of junctional plaque proteins and are involved in cell-matrix adhesions (Lo, 2006). The regulators of cell motility in these structures are Rho and Cdc42 family members (Jiang et al., 2009). The actin machinery is involved as well in the organization of invadopodia and podosomes, actin-rich membrane protrusions that, similar to focal adhesions, are features related to invasiveness in cancer (Albiges-Rizo et al., 2009).

We observed the up-modulation of other relevant gene families, i.e., cytokine-cytokine receptor interaction: CCL26 (chemokine, C-C motif, ligand 26), CCR7 (chemokine, C-C motif, receptor 7), CLCF1 (cardiotrophin-like cytokine factor 1), TNFRSF21 (tumor necrosis factor receptor superfamily, member 21), IL1RAP (interleukin 1 receptor accessory protein), IL8 (interleukin 8), IL11 (interleukin 11), INHBB (inhibin, beta B), LIF (leukemia inhibitory factor), MET (met proto-oncogene), TNFRSF12A (tumor necrosis factor receptor superfamily, member 12A), IL23A (interleukin 23, alpha subunit), TGFBR2 (transforming growth factor, beta receptor II), CXCR4 (chemokine, C-X-C motif, receptor 4), RELT (RELT tumor necrosis factor receptor), TNFRSF25 (tumor necrosis factor receptor superfamily, member 25) and OSMR (oncostatin M receptor); classical MAPKinase: MAP3K14 (mitogen-activated protein kinase 14), RELB (v-rel reticuloendotheliosis viral oncogene homolog B), DUSP6 (dual specificity phosphatase 6), DUSP1 (dual specificity phosphatase 1), DUSP4 (dual specificity phosphatase 4), DUSP9 (dual specificity phosphatase 9), FOS (FBJ murine osteosarcoma viral oncogene homolog), and JNK-p38; MAPKinase pathway: GADD45G (growth arrest and DNA-damage-inducible, gamma), TGFBR2 (transforming growth factor, beta receptor II), RAC2 (ras-related C3 botulinum toxin substrate 2), PAK1 (p21 protein activated kinase 1), MAP3K6 (mitogen-activated protein kinase kinase kinase 6), DUSP1, DUSP4, DUSP9, JUN, GADD45B (growth arrest and DNA-damage-inducible, beta), HSPA1A (heat shock $70 \mathrm{kDa}$ protein $1 \mathrm{~A}$ ), HSPA6 (heat shock $70 \mathrm{kDa}$ protein 6 ) and NR4A1(nuclear receptor subfamily 4, group A, member 1); and finally cancer: BIRC3 (baculoviral IAP repeat-containing 3) and RAC2 (ras-related C3 botulinum toxin substrate 2) involved in evading apoptosis, WNT6 (wingless-type MMTV integration site family, member 6), SLC2A1 (solute carrier family 2, member 1 and IL8 sustaining angiogenesis, JUN (jun proto-oncogene), FOS, MMP1 (matrix metallopeptidase 1), TGFBR2 (transforming growth factor, beta receptor II) inducing insensitivity to antigrowth signal; GSTP1 (glutathione S-transferase pi 1) involved in carcinogens damage to DNA. 
On the other hand, we found a down-modulation of the p53 signaling pathway: CDKN1A (cyclin-dependent kinase inhibitor 1A), GADD45G (growth arrest and DNA-damage-inducible, gamma), GADD45B (growth arrest and DNA-damageinducible, beta), SERPINE1 (serpin peptidase inhibitor, clade E member 1).

To go deeply into the mechanisms of Cd toxicity and carcinogenicity, we analyzed the expression of miRNAs. Vital biological processes including differentiation, proliferation, and apoptosis are controlled by miRNAs, non-coding regulatory RNA molecules that bind target mRNA, thus suppressing their translation into proteins. Alterations in miRNAs function and regulation have emerged as key factors in cancer (Ventura and Jacks, 2009). In our study, the main percentage of miRNAs regulated by $\mathrm{Cd}$ exposure belongs to the let- 7 family. Let-7 has been described as a tumor suppressor miRNA, and, as observed, these family members are down-regulated in many cancers when normal tissues are compared to cancer counterparts (Boyerinas et al., 2010). In some tumors all the let-7 members are down-regulated, whereas in some cancers only specific members are down-regulated. In a study on miRNAs toxicogenomics (Izzotti et al., 2011), the eight let-7 miRNAs are down-regulated in rat lung exposed to cigarette smoke, the only exception being let7-f.

It was also demonstrated that human let-7 does specifically target RAS in human cancer cells. This direct targeting of RAS by let-7 was confirmed in non-small cell lung cancer (NSCLC), where, in a murine model, let-7g inhibited tumor growth via suppression of RAS (Kumar et al., 2008). Furthermore, mir$15 \mathrm{~b}$, another miRNA down-regulated in our study, has been described as a tumor suppressor because it regulates apoptosis by targeting Bcl-2 (Guo et al., 2009).

In the analysis of enrichment in KEGG pathways of the downregulated miRNAs targets, the two top pathways were focal adhesion (hsa04510) and MAPK signaling pathway (hsa04010) (Tab. S3, supplementary file at www.altex-edition.org). The involvement of focal adhesions in cancer-related mechanisms has been described above. The mitogen-activated protein kinase (MAPK) cascade is a highly conserved module that is involved in various cellular functions, including cell proliferation, differentiation and, migration. These two pathways are important in regulation processes that, when malfunctioning, can promote cancer development. As shown in Table S3, all of the ten most enriched pathways are involved in cancer (Huang et al., 2010).

Intriguingly, in the analysis of gene expression data for enrichment in KEGG pathways of up-regulated genes, we identified an overlap with the KEGG enrichment of predicted target of down-regulated miRNAs target. The first pathway is the same for both analyses (focal adhesion, Fig. 4) and the second of miRNAs target is the fourth in mRNA analysis (MAPK signaling pathway). From this we can argue that miRNAs probably have a role in the regulation of those pathways.

In the future, the characterization of miRNAs and mRNAs interaction will provide better insight into $\mathrm{Cd}$ mechanisms. So far, the data presented in this paper show that miRNAs are potentially involved in the regulation induced in the cell by $\mathrm{Cd}$, and they can help provide a better understanding of the mechanism of action of this metal. In addition, our data, along with recent papers from the literature (Izzotti et al., 2011), gives evidence that the complexity of the molecular machinery involved in $\mathrm{Cd}$ toxicity and carcinogenicity is far from being completely elucidated. Finally, even though the human HepG2 cell line is a useful in vitro model for Cd toxicity studies (Dehn et al., 2004; Urani et al., 2005, 2007, 2010), the future characterization of human primary hepatocytes in response to $\mathrm{Cd}$ exposure will provide information on normal cells to be compared to HepG2.

\section{References}

Albiges-Rizo, C., Destaing, O., Fourcade, B., et al. (2009). Actin machinery and mechanosensitivity in invadopodia, podosomes and focal adhesions. J. Cell Sci. 122, 3037-3049.

Andrew, A. S., Warren, A. J., Barchowsky, A., et al. (2003). Genomic and proteomic profiling of responses to toxic metals in human lung cells. Environ. Health Perspect. 111, 825-835.

Ao, L., Liu, J. Y., Liu, W. B., et al. (2010). Comparison of gene expression profiles in BALB/c $3 \mathrm{~T} 3$ transformed foci exposed to tumor promoting agents. Toxicol. In Vitro 24, 430-438.

Badisa, V. L., Latinwo, L. M., Odewumi, C. O., et al. (2008). Cytotoxicity and stress gene microarray analysis in cadmiumexposed CRL-1439 normal rat liver cells. Int. J. Mol. Med. 22, 213-219.

Bartel, D. P. (2009). MicroRNAs: target recognition and regulatory functions. Cell 136, 215-233.

Bertin, G. and Averbeck, D. (2006). Cadmium: cellular effects, modifications of biomolecules, modulation of DNA repair and genotoxic consequences (a review). Biochimie 88, 1549-1559.

Bonham, R. T., Fine, M. R., Pollock, F. M., et al. (2003). Hsp27, Hsp70, and metallothionein in MDCK and LLC-PK1 renal epithelial cells: effects of prolonged exposure to cadmium. Toxicol. Appl. Pharmacol. 191, 63-73.

Boyerinas, B., Park, S. M., Hau, A., et al. (2010). The role of let7 in cell differentiation and cancer. Endocr. Relat. Cancer 17, F19-36.

Dakeshita, S., Kawai, T., Uemura, H., et al. (2009). Gene expression signatures in peripheral blood cells from Japanese women exposed to environmental cadmium. Toxicology 257, 25-32.

Dehn, P. F., White, C. W., Conners, D. E., et al. (2004). Charatcterization of the human hepatocellular carcinoma (HepG2) cell line as an in vitro model for cadmium toxicity studies. In Vitro Cell Dev. Biol. Anim. 40, 172-182.

Edgar, R., Domrachev, M., and Lash, A. E. (2002). Gene Expression Omnibus: NCBI gene expression and hybridization array data repository. Nucleic Acids Res. 30, 207-210.

Gentleman, R. C., Carey, V. J., Bates, D. M., et al. (2004). Bioconductor: open software development for computational biology and bioinformatics. Biol. 5, R80.

Guo, C.-J., Pan, Q., Li, D.-G., et al. (2009). miR-15b and miR-16 are implicated in activation of the rat hepatic stellate cell: An essential role for apoptosis. J. Hepatol. 50, 766-778.

Hartwig, A., Asmuss, M., Blessing, H., et al. (2002). Interference by toxic metal ions with zinc-dependent proteins involved in maintaining genomic stability. Food Chem. Toxicol. 40, 11791184. 
Hartwig, A. (2010). Mechanisms in cadmium-induced carcinogenicity: recent insights. Biometals 23, 951-960.

Honda, A., Komuro, H., Nagase, H., et al. (2010). Microarray analysis of the liver in metallothionein-III null mice treated with cadmium. J. Toxicol. Sci. 35, 271-273.

Huang, D. W., Sherman, B. T., and Lempicki, R. A. (2009). Systematic and integrative analysis of large gene lists using DAVID bioinformatics resources. Nat. Protoc. 4, 44-57.

Huang, P., Han, J., and Hui, L. (2010). MAPK signaling in inflammation-associated cancer development. Protein Cell 1, 218226.

IARC (1993). Meeting of the IARC working group on beryllium, cadmium, mercury and exposures in the glass manufacturing industry. Scand. J. Work Environ. Health 19, 360-363.

Izzotti, A., Larghero, P., Longobardi, M., et al. (2011). Dose-responsiveness and persistence of microRNA expression alterations induced by cigarette smoke in mouse lung. Mutat. Res. 717, 9-16.

Jiang, P., Enomoto, A., and Takahashi, M. (2009). Cell biology of the movement of breast cancer cells: intracellular signalling and the actin cytoskeleton. Cancer Lett. 284, 122-130.

Kawata, K., Shimazaki, R., and Okabe, S. (2009). Comparison of gene expression profiles in HepG2 cells exposed to arsenic, cadmium, nickel, and three model carcinogens for investigating the mechanisms of metal carcinogenesis. Environ. Mol. Mutagen. 50, 46-59.

Klaassen, C. D., Liu, J., and Diwan, B. A. (2009). Metallothionein protection of cadmium toxicity. Toxicol. Appl. Pharmacol. 238, 215-220.

Koizumi, S. and Yamada, H. (2003). DNA microarray analysis of altered gene expression in cadmium-exposed human cells. $J$. Occup. Health 45, 331-334.

Kumar, M. S., Erkeland, S. J., Pester, R. E., et al. (2008). Suppression of non-small cell lung tumor development by the let-7 microRNA family. Proc. Natl. Acad. Sci. USA 105, 3903-3908.

Lambrechts, A., Van Troys, M., and Ampe, C. (2004). The actin cytoskeleton in normal and pathological cell motility. Int. J. Biochem. Cell Biol. 36, 1890-1909.

Li, Y., Kimura, T., Huyck, R. W., et al. (2008). Zinc-induced formation of a coactivator complex containing the zinc-sensing transcription factor MTF-1, p300/CBP, and Sp1. Mol. Cell Biol. 28, 4275-4284.

Lo, S. H. (2006). Focal adhesions: what's new inside. Dev. Biol. 294, 280-291.

Maragkakis, M., Reczko, M., Simossis, V. A., et al. (2009). DIANA-microT web server: elucidating microRNA functions through target prediction. Nucleic Acids Res. 37, W273-276.

Martelli, A., Rousselet, E., Dycke, C., et al. (2006). Cadmium toxicity in animal cells by interference with essential metals. Biochimie 88, 1807-1814.

Ohana, E., Sekler, I., Kaisman, T., et al. (2006). Silencing of ZnT-1 expression enhances heavy metal influx and toxicity. Mol.Med. 84, 753-763.

Papadopoulos, G. L., Alexiou, P., Maragkakis, M., et al. (2009). DIANA-mirPath: Integrating human and mouse microRNAs in pathways. Bioinformatics 25, 1991-1993.
Rao, J. Y., Hurst, R. E., Bales, W. D., et al. (1990). Cellular F-actin levels as a marker for cellular transformation: relationship to cell division and differentiation. Cancer Res. 50, 2215-2220.

Satarug, S. and Moore, M. R. (2004). Adverse health effects of chronic exposure to low-level cadmium in foodstuffs and cigarette smoke. Environ. Health Perspect. 112, 1099-1103.

Smyth, G. K. (2004). Linear models and empirical bayes methods for assessing differential expression in microarray experiments. Stat. Appl. Genet. Mol. Biol. 3, Article3.

Talio, M. C., Luconi, M. O., Masi, A. N., et al. (2010). Cadmium monitoring in saliva and urine as indicator of smoking addiction. Sci. Total Environ. 408, 3125-3132.

Urani, C., Melchioretto, P., Canevali, C., et al. (2005). Cytotoxicity and induction of protective mechanisms in HepG2 cells exposed to cadmium. Toxicol In Vitro. 19, 887-892.

Urani, C., Melchioretto, P., Canevali, C., et al. (2007). Metallothionein and hsp70 expression in HepG2 cells after prolonged cadmium exposure. Toxicol. In Vitro 21, 314-319.

Urani, C., Melchioretto, P., and Gribaldo, L. (2010). Regulation of metallothioneins and ZnT-1 transporter expression in human hepatoma cells HepG2 exposed to zinc and cadmium. Toxicol. In Vitro 24, 370-374.

Ventura, A. and Jacks, T. (2009). MicroRNAs and cancer: short RNAs go a long way. Cell 136, 586-591.

Waalkes, M. P. (2003). Cadmium carcinogenesis. Mutat. Res. 533, 107-120.

\section{Acknowledgments}

C.U. gratefully acknowledges the partial support by Fondo di Ateneo per la Ricerca, granted by Università di Milano Bicocca and the European Commission.

\section{Correspondence to}

Marco Fabbri

European Commission - Joint Research Centre

Institute for Health and Consumer Protection

Molecular Biology and Genomics unit

TP 464, Via E. Fermi, 2749

21027 Ispra (VA)

Italy

Phone: +390332789538

Fax: +390332785446

e-mail: marco.fabbri@jrc.ec.europa.eu

Chiara Urani

Dipartimento di Scienze dell'Ambiente e del Territorio

Università degli Studi di Milano Bicocca

Piazza della Scienza, 1

20126 Milano

Italy

Phone: +390264482923

Fax +390264482996

e-mail: chiara.urani@unimib.it 